\title{
Política de la responsabilidad: Desde Hans Jonas hacia Iris Marion Young
}

\author{
Responsibility Policy: From Hans Jonas \\ to Iris Marion Young
}

ERIC POMMIER (iD)

\section{Resumen}

En este artículo, quisiera mostrar, en un primer momento, en qué sentido se puede decir que el sujeto de la responsabilidad en Jonas es problemático. Esta dimensión problemática del sujeto ético jonasiano abre, en un segundo momento, a una reflexión sobre la manera de desidealizarlo, hacerlo salir de una cierta abstracción gracias a consideraciones de carácter materialista tomando en cuenta la infraestructura económica de la sociedad. Sin embargo, en un último momento, trataré de mostrar que no basta con esta concepción material de la subjetividad para concretizarla y hacer efectiva la toma de responsabilidad hacia la Tierra y las generaciones futuras. Se requiere una concepción compartida de la responsabilidad lo que presupone una visión relacional de la sociedad tal como Iris Marion Young la propone.

Palabras-clave: Economía. Marxismo. Política. Responsabilidad. Técnica.

\begin{abstract}
In the first place, I would like to show how we can say that the subject of responsibility is problematic in the frame of Hans Jonas's thinking. In the second place, the problematical status of the jonasian ethical subject opens to meditation about the means of making it more concrete thanks to materialistic analysis that take into account the economic infrastructure of the society. To conclude, we will see that a materialistic conception of the subject is not sufficient to make effective our responsibility towards the Earth and the Future Generations. We need to
\end{abstract}

\footnotetext{
a Pontificia Universidad Católica de Chile, Santiago, Chile. Doutor em Filosofia (Paris Sorbonne I), email: espommier@gmail.com
} 
promote the model of a shared responsibility, which relies on a "social connexion" of the society as the one raised by Iris Marion Young.

Keywords: Economy. Marxism. Politics. Responsibility. Technique.

En este artículo, quisiera mostrar, en un primer momento, en qué sentido se puede decir que el sujeto de la responsabilidad en Jonas es problemático. Esta dimensión problemática del sujeto ético jonasiano abre, en un segundo momento, a una reflexión sobre la manera de desidealizarlo, hacerlo salir de una cierta abstracción gracias a consideraciones de carácter materialista tomando en cuenta la infraestructura económica de la sociedad. Sin embargo, en un último momento, trataré de mostrar que no basta con esta concepción material de la subjetividad para concretizarla y hacer efectiva la toma de responsabilidad hacia la Tierra y las generaciones futuras. Se requiere una concepción compartida de la responsabilidad lo que presupone una visión relacional de la sociedad tal como Iris Marion Young la propone.

\section{La técnica moderna y el sujeto de la responsabilidad según Jonas.}

El interés de Hans Jonas por la formulación y la fundamentación de una nueva ética adaptada a la civilización contemporánea procede de una reflexión sobre los desafíos axiológicos y deontológicos planteados por la técnica moderna. Si ésta se caracteriza por un tipo de actuar que podría comprometer la vida humana como humana y la naturaleza, entonces hay que promover un tipo de actuar alternativo capaz de preservar esta vida y esta naturaleza. La directriz del Principio Responsabilidad plantea la pregunta por la praxis ética de manera que ella pueda contrastar y reorientar la acción técnica. Pero, ¿en qué medida se puede decir que la técnica requiere ahora una nueva ética? ¿Qué tienen de nuevo las nuevas técnicas para requerir una ética nueva? Al respecto, en el segundo capítulo de Técnica, Medicina y Ética, Hans Jonas enfatiza al menos tres características dignas de consideración.

En primer lugar, la técnica se vuelve ambivalente. Mientras la técnica tradicional podía ser vista como axiológicamente neutra, sucede ahora que por sí misma ella puede desarrollarse de manera perjudicial para la humanidad. Ya no se puede decir que la 
evaluación moral de la técnica depende del fin al servicio del cual ella se pone. Ya no se puede decir que la técnica es solo un medio al servicio de un fin que puede ser bueno o malo. En efecto, el uso intencionalmente bueno de una técnica puede ser malo respecto de sus consecuencias. Puede ser bueno que el padre lleve en auto a su hijo a la escuela por la mañana. Sin embargo, la acumulación de estas buenas acciones a nivel planetario aumenta la contaminación y el calentamiento global. Este comentario conduce directamente al segundo rasgo de la técnica moderna. Es necesario reconocer que ella tiene una envergadura planetaria tanto a nivel espacial como a nivel temporal. El uso de la técnica es cada vez menos circunscrito a una región del mundo. Lo que se hace aquí y ahora tiene, cada vez más y en virtud del carácter acumulativo de la técnica, efectos a nivel del sistema Tierra y un impacto sobre las condiciones de vida de las generaciones futuras.

Por último, no se puede desconocer el carácter cada vez menos libre del desarrollo técnico. Mientras la naturaleza era el lugar de la necesidad y la técnica el poder específicamente humano gracias al cual él podía introducir contingencia en ella, sucede ahora que es la naturaleza la que se vuelve contingente y vulnerable - su existencia está puesta en cuestión - y la técnica la que obedece a la necesidad. Su desarrollo presenta un carácter casi fatal. El ser humano tiene cada vez menos distancia con ella. El plazo entre la capacidad y el uso, entre la posibilidad y la práctica se achica. Si bien, por ejemplo, de la capacidad de hablar no se deriva la decisión de usarla, todo pasa como si ahora hubiera ahora una vitalización de un carácter particular de la sociedad: de la misma manera en que el poder respirar tiene que actualizarse para que el organismo siga viviendo, los poderes técnicos de una sociedad tienen que actualizarse para que ella pueda seguir asegurando sus necesidades que le aparecen como siendo vitales.

La conjugación de la primera y tercera característica es muy temible. El uso implacable e intencionalmente loable de la técnica puede generar catástrofes cuya peligrosidad es la lentitud de su desarrollo. La ventaja de las catástrofes del tipo producidas por el uso de las bombas nucleares proviene del carácter inmediatamente visible de su impacto destructivo. Les hace identificables e intimidantes de tal manera, que se las pueda evitar. Al contrario, las bombas ecológicas producidas por el calentamiento global o el envenenamiento del suelo sacan su carácter peligroso de su dimensión poco visible en el presente, de su carácter progresivo y a priori bueno de las intenciones en juego (uso del auto para ir al trabajo, abonos químicos, energía nuclear para proporcionar luz a las ciudades). 
Como escribe Jonas: "Mientras el mal hermano Caín - la bomba - yace encadenado en su cueva, el buen hermano Abel - el pacífico reactor - sigue sin dramatismo depositando su veneno para futuros milenios" (JONAS, 1997, p. 37).

Hans Jonas identifica acá, con una cierta lucidez, los efectos del desarrollo unilateral de la razón técnica, o calculante, que manda el proceso de mundialización a causa del cual todo, ser natural, vivo o humano, puede ser objetivado y reconfigurado a gusto. La tecnificación del mundo no tiene solo que ver con el aumento de los objetos artificiales o con la valoración de los saberes prácticos sino con el monopolio otorgado a un cierto tipo de racionalidad: la racionalidad instrumental. Efectivamente, como Hans Jonas ya lo había analizado en The Phenomenon of Life, cambió de manera esencial la forma del saber. En lugar de contemplar las Ideas que podían ser objetos de placer y de consideración por sí misma en la filosofía de Platón, el saber saca ahora su legitimidad de su fecundidad práctica. Todavía más; el mismo saber se vuelve un tipo de refabricación del objeto considerado. La concepción del saber que prevale es una concepción técnica (JONAS, 2001, p. 204). Desaparece la idea de un fin en sí, de un fin último que pueda guiar el desarrollo técnico hacia una finalidad de verdad. Este mismo se vuelve el fin en sí, pero un fin incapaz de guiar el desarrollo histórico del ser humano. El devenir se vuelve más automático que propiamente histórico. La mundialización hecha posible por la tecnificación del mundo nos hizo entrar en el nihilismo, en un mundo - si es un mundo - sin sentido.

Sin embargo, este diagnóstico no invita a la desesperación, a la resignación o a la aceptación pasiva. Al contrario del carácter casi-fatal del desarrollo unilateralmente técnico (Jonas escribe "fast wie ein Schicksal" (JONAS, 1984, p. 388), hay que promover un desarrollo histórico ético. A contra de la mundialización, hay que mundanizar y, por ello, se requiere una responsabilidad de carácter cósmico (JONAS, 1997, p. 36). Efectivamente, la ética ineficiente de la intención hay que substituirla por una ética de la responsabilidad que pueda controlar lo que nos controla gracias a una evaluación de los efectos de nuestra acción tecnológica con el fin de pararlos u de orientarlos éticamente.

Ya que lo que está en juego con la hipertrofia de la técnica contemporánea no es otra cosa que el desarrollo de la vida propiamente humana en su conjunto, la naturaleza, hasta la misma Tierra, es necesario reconocer que el imperativo ético de Jonas -en la fundamentación ontológica de la cual no entro aquí (JONAS, 1984, p. 61-242; POMMIER, 2013, p. 137-169, p. 203-223) - se dirige hacia la permanencia de una vida auténticamente 
humana, es decir una vida que sea capaz de responsabilidad con la vida en su conjunto. El objeto de la responsabilidad en Jonas no se limita a las generaciones humanas futuras sino también a la vida como tal. La vida tiene un valor en sí y tiene que ser respetada de modo que la destrucción innecesaria de una especie puede ser asimilada a un crimen en sí. Como dice Jonas en Técnica, Medicina y Ética: "toda extinción de especies arbitraria e innecesaria se convierte en crimen en sí misma.” (JONAS, 1997, p. 36)

Ahora bien, ¿quién es exactamente el sujeto de responsabilidad? Puesto que solo el ser humano es capaz de destrucción generalizada y puesto que él solo puede hacerse receptivo al deber de responsabilidad, él solo tiene esta responsabilidad. Dicho de otro modo, ya que está en una situación en la cual tiene la responsabilidad de lo que está pasando, él debe tomarla. No es porque no la tome que deja de tenerla. Puede huir de esta responsabilidad, hacer oídos sordos a su llamada, esconderse a sí mismo la angustia ética que le asalta cuando empieza a hacerse presente su responsabilidad frente al desarrollo ecohumano. Tal huida no le evita nada a su ser constitutivamente responsable de la situación contemporánea de crisis ecológica.

Sin embargo, la comprensión jonasiana del sujeto de responsabilidad sigue siendo bastante enigmática. Efectivamente, si el problema planteado por la técnica es el de la acumulación nociva de sus efectos perniciosos y el carácter automático de su desarrollo, no se ve bien cómo se podría limitar o parar la máquina infernal que conduce a la autodestrucción. Es la razón por la cual Jonas nos avisa que la ética de la responsabilidad concierne al actor público, al poder político (JONAS, 1984, p. 32; DAVID, RÖPCKE, 2004, p. 209). Solo una acción de carácter colectivo puede tener una eficacia ética. Sin embargo, no se ve bien acá qué política podría realizar el principio ético. No se ve bien cuál es la concepción que Jonas tiene del sujeto político. ¿Cómo se debe pensar la organización social y política para que haga posible el cumplimiento del principio de responsabilidad?

\section{¿Materializar la responsabilidad?}

Por falta de una reflexión más acabada sobre lo político, la filosofía practica de Jonas corre el riesgo de un cierto idealismo. Puede, incluso, dar lugar a la sospecha de un uso ideológico de la ética. Efectivamente, al enfocarse sobre la necesidad de una responsabilidad 
en general hacia la humanidad en general, al promover un deber abstracto que cuenta con la buena voluntad de los agentes para que ellos tomen en consideración las consecuencias de su actuar técnico, pasa por alto (o se disimula) el hecho de que la crisis ecológica procede de un cierto tipo de estructuración de la base económica de la sociedad caracterizada por la oposición, violenta pero escondida, de intereses materiales que ponen en juego el sistema de producción y de consumo. Es así como la llamada a la responsabilidad ética podría ser vista como la máscara de un poder, esencialmente económico. Este poder buscaría esconderse de serlo y disimular las ventajas materiales que saca del uso de tal poder. Fuera de las apariencias, fuera de las representaciones o ilusiones éticas, dicho de otro modo, fuera de una superestructura ideológica, se podría desvelar la realidad de una explotación de la Tierra, de una parte de la humanidad y de los seres vivos, por otra parte de la humanidad, la que tiene el control, la propiedad privada de los medios de producción. Solo un análisis de la infraestructura socio-económica hace posible, por una parte, la identificación de las verdaderas víctimas y verdaderos responsables de la crisis ecológica, por otra, la determinación real de las causas del derrumbamiento global (MALM, 2017, p. 7-17). No es el ser humano en general o la Humanidad la que tiene que hacerse responsable de la preservación de la humanidad por medio de la inteligencia y de la buena voluntad. Son las víctimas del sistema de producción y de consumo -el proletariado- los que tienen que entrar en una lucha para hacer valer sus intereses y desprivatizar el sistema de producción que conduce al saqueo de la Tierra. Si bien se dice hoy en día, bajo la denominación de Antropoceno (CRUTZEN, 2002), que hemos entrado en una nueva época geológica en la cual el ser humano se hizo fuerza geológica capaz de modificar de manera substancial el equilibrio planetario (ya no hay separación entre la historia y la naturaleza), es necesario reconocer que tal denominación es incorrecta e ideológica. Ella prepara las mentes a una posible gestión meramente técnico-científica de la crisis ecológica, que perpetua la dominación de una parte de la humanidad sobre la otra, una sola teniendo los recursos económicos para protegerse del desastre provocado por su auri sacra fames. Sería mejor hablar del Capitaloceno para enfatizar el carácter material del problema (ULLOA, 2018, p. 284-288). Solo una teoría de las relaciones sociales de carácter marxista parece permitir desidealizar el principio responsabilidad y volver a las verdaderas causas (materiales) de las catástrofes medioambientales. 
Por supuesto, se podría dudar de que una lectura marxista de la realidad social nos de ojos para interpretar y superar la crisis ecológica. Efectivamente, la aspiración a una revolución que haga posible la instauración de la justicia social parece dejar afuera del campo de las preocupaciones posibles el cuidado de la naturaleza. El enfoque marxista parece ser bastante antropocéntrico. Se podría incluso decir que la realización de la justicia interhumana tiene que pasar por un hiperdesarrollo de la técnica que empeoraría la situación medioambiental del planeta (JONAS, 1984, p. 327-337, p. 370). Para que cada uno pueda disfrutar de "los frutos de la Tierra" en función de su necesidad, es necesario seguir manteniendo un alto nivel de producción. La naturaleza sigue siendo concebida como conjunto de recursos. Sigue siendo el lugar de una instrumentalización. Si bien su apropiación podría dejar de ser capitalista, ella seguirá siendo objeto de una apropiación por medio de la técnica. Bajo este aspecto, el marxismo no conduciría a desidealizar el principio responsabilidad sino seria él mismo una visión utópica del mundo, alimentada por un movimiento de reconfiguración total de la naturaleza por medio de una tecnologización unilateral del mundo (JONAS, 1984, p. 388).

De hecho, es precisamente esta lectura que Jonas hace del marxismo en la última parte del Principio Responsabilidad, cuando examina qué tipo de "régimen político" podría concretizar el principio responsabilidad. Lejos de que el marxismo nos ofrezca los recursos teóricos y prácticos para movilizar los sujetos hacia la conversión ecológica y para encarnar el principio responsabilidad, él no es otra cosa que la expresión de una utopía de reconfiguración total y técnica del mundo que tiene que ser criticada considerando al principio de responsabilidad (OLIVEIRA, POMMIER, 2019, p. 139-143). En ese sentido, esta utopía heredaría del dualismo cartesiano que pretende hacerse dueño de la naturaleza al imponerle la ley de la subjetividad y, por lo que nos concierne, la ley de la razón instrumental. El ser humano accede a la libertad a través de un proceso de domesticación de la naturaleza que se puede asemejar a un proceso de reconfiguración de la naturaleza. Solo así podríamos emanciparnos del reino de la necesidad natural en beneficio de una libertad pura gracias a la cual el ser humano puede realmente ser sí mismo, en particular a través de un proceso intersubjetivo de reconocimiento recíproco de las subjetividades libres (JONAS, 1984, p. 343-348).

No obstante, no se puede desconocer los esfuerzos del marxismo ecológico encarnados por ejemplo por John Bellamy Foster y Jason Moore para compatibilizar el 
marxismo con la ecología, hacer evolucionar el marxismo hacia la ecología y quitarle a la ecología su dimensión "moralizadora” de "alma bella”. Es así como Bellamy Foster tematizará, a partir de Marx, la noción de ruptura del metabolismo social al mostrar cómo el modo capitalista de organización de la labor conduce a perturbar el equilibrio de los intercambios entre la sociedad y la naturaleza. El modo de apropiación capitalista hace insustentable la relación dinámica entre Tierra y Humanidad, por ejemplo porque la agricultura intensiva y mecanizada orientada hacia el provecho ciego provoca el empobrecimiento en nutrientes de los suelos, principalmente en los países de la "periferia", y a una exportación de la riqueza de los suelos hacia los países del "centro" (GUILLIBERT; HABER, 2017, p. 15). Acá aparece que el modo de organización capitalista de la producción, un modo de organización social entre otros a lo largo de la historia, favorece e intensifica la crisis ecológica. Por su lado, Jason Moore enfatiza la tendencia del capitalismo a sacar provecho del trabajo de la naturaleza (la energía acumulada en los suelos, las materias primarias, la alimentación) para apropiárselo, sin compensación (MOORE, 2017, p. 34, 45), usarlo para aumentar sus intereses privados y, así, seguir reduciendo los recursos de una Tierra que son limitados mientras las aspiraciones al lucro del capitalismo son ilimitadas.

Desde este punto de vista, no habría que llamar a la responsabilidad de todos, pues los verdaderos responsables de la crisis son solamente los capitalistas. Tampoco habría que contar con el Estado y sus instituciones pues no hacen otra cosa que expresar los intereses de la clase dominante. El Estado es la expresión del derecho de lo más económicamente fuerte, la burguesía (MARX, 1966, p. 36). Solo el fomento de una lucha de clases a nivel social, que pudiera dar lugar a la superación de la división social en beneficio de un régimen político que cancelaría el perseguimiento del lucro y la explotación humana, permitiría desembocar en un sistema de gestión de los recursos orientados hacia la satisfacción de las necesidades de cada uno sin sobreexplotar los recursos naturales y el trabajo no remunerado de la naturaleza. Para que no se derrumbe el sistema Tierra, se debe derrumbar el sistema capitalista (MOORE, 2017, p. 45).

Sin embargo, creo que podemos dudar del carácter realmente transformador de esta concepción de lo social. En primer lugar, si bien el capitalismo acelera, intensifica, dramatiza la crisis ecológica que tenemos que enfrentar, es necesario reconocer también que la tecnificación del mundo y sus derivas parecen ser irreductibles a la sola lógica capitalista de modo que el desarrollo alternativo o comunista no ofrece, con plena certeza, las garantías 
deseadas para que haya una evolución sustentable de las relaciones entre los seres humanos y la naturaleza. No va de suyo, por un lado, que la hipertecnificacion del mundo solo procede de una búsqueda de lucro por una parte de la humanidad y, por otro lado, que un régimen comunista preocupado por la igualdad de condiciones pueda alcanzar esta meta sin contar con un desarrollo hiperbólico del poder técnico. En segundo lugar, podríamos preguntarnos por saber si resulta conveniente oponer de manera tan drástica la clase de los explotados y la de los explotadores. En efecto, si es evidente que los más pobres sufren más y son más expuestos a la degradación ambiental que los más ricos, es cierto también que los más ricos también son expuestos a la perspectiva de la catástrofe ecológica (CHAKRABARTY, 2018, p. 158-162). Existen desigualdades ambientales pero la destinación de la humanidad conserva un horizonte común. Es una misma humanidad que tendrá y tiene que enfrentar la adversidad ecológica. Por otro lado, no queda claro cómo se podría transformar una realidad social caracterizada por su injusticia sistémica. Tenemos la impresión de que el marxismo es el lugar de una ambigüedad. Por un lado, apunta a un sistema de relaciones causales que objetiva las relaciones humanas, quita a los agentes cualquier libertad, hace de la revolución un efecto necesariamente determinado por las contradicciones del sistema capitalista. Por otro lado, llama a la toma de conciencia de la explotación, a la constitución del proletariado en clase social capaz de representarse el origen de su alienación y su función de agente transformador del sistema social (CHÂTELET, 1986, p. 99-101). El sujeto parece ser a la vez una mera cosa desprovista de iniciativa e incapaz de transformar la realidad y un agente absolutamente libre que puede transformar la totalidad social por el solo esfuerzo de la toma de conciencia del sistema que le explota. Por un lado, el sujeto es el mero resultado de una causalidad social que le determina por completo. Por otro lado, él es quien tiene que construir esta causalidad, erigirse en sujeto de conocimiento científico de lo social (MERLEAU-PONTY, 1965, p. 124-125). En ambos casos, que él sea el Sísifo condenado a empujar cuesta arriba por la montaña social su piedra o el Prometeo que trastorna de forma radical el todo social, no se entiende bien cómo se podría pensar una verdadera teoría de la transformación social capaz de encarnar el principio responsabilidad.

\section{El "social connection model" de Iris Marion Young.}


Estas meras dudas no deben conducir a desconocer la importancia del factor económico al momento de pensar la organización social de manera que ella sea compatible con una responsabilidad hacia la naturaleza y las generaciones futuras. En efecto, en primer lugar, el miembro de la sociedad pertenece a una situación económica específica. Se podría agregar que pertenece a una situación socio-ambiental específica. Esta inscripción en una situación eco-ambiental específica no determina unilateralmente su comportamiento y sus representaciones. Él no es el producto de esta situación que actuaría sobre él como una causa mecánica. Pero tampoco sus acciones y sus representaciones son desvinculadas de esta situación, pues ésta pre-determina el campo de sus posibilidades de actuar y de pensar. En segundo lugar, su posición en el espacio eco-ambiental le expone o no a una vulnerabilidad eco-ambiental. Si seguimos los análisis de Iris Marion Young en Responsibility for Justice, debemos reconocer que el hecho de ocupar una posición de vulnerabilidad no es justo, pues este hecho no se puede explicar por el mero azar, las decisiones individuales, la sola lógica del mercado o las políticas estatales (YOUNG, 2013, p. 45-52). Dicho de otro modo, existe una injusticia estructural que no procede por sí sola de las políticas públicas o de las interacciones individuales, sino del conjunto de todos estos factores. La pérdida de libertad de los pájaros encerrados en una jaula no se debe a una de las barras de metal de la jaula sino a su suma. Así es como el agente social puede sentirse atrapado en una posición ecoambiental que le hace más vulnerable que otros. El punto que Young enfatiza es que, a diferencia de lo que piensa John Rawls (YOUNG, 2013, p. 70), el origen de la "alienación" no se encuentra en una parte de la sociedad en específico (las leyes del mercado, las relaciones familiares, el sistema escolar etc.) sino en el modo de organización en su conjunto, a la estructura de la sociedad en su totalidad, a una cierta manera de representársela. Esta consideración no conduce a abandonar la perspectiva del progreso social como si hubiera un fatalismo de la lógica sistémica pues, como lo veremos, esta visión de la realidad social invita claramente a desdoblar nuestra mirada sobre ella, por un lado, apuntando a la manera con la cual tratamos las personas de nuestro entorno, por otro, considerando la manera con la cual perpetuamos o no una injusticia estructural en función de la posición eco-ambiental que ocupamos y de nuestro actuar, y ello aún cuando tratamos de forma decente y amable las personas de nuestro entorno.

Es así como la toma de conciencia de la existencia de la injusticia estructural a nivel social es, para Young, el punto de partida de una conciencia de responsabilidad de carácter 
politico 1 . En efecto, el mero hecho de pertenecer a un sistema social que produce injusticia hace de nosotros, al menos cada vez que actuamos y actualizamos o reproducimos la estructura social, personas que tienen una responsabilidad en la perpetuación de la injusticia. Desde este punto de vista, no escapamos de la responsabilidad pues somos miembros necesariamente activos del sistema social del cual recibimos ventajas. Sin embargo, tener una responsabilidad no quiere decir todavía tomar esta responsabilidad. De hecho, como lo veremos con Young, existe la tentación de huir de la toma de responsabilidad que, no obstante, tenemos. Inspirándose de Arendt, Young hace valer que la dimensión política de esta responsabilidad procede de su carácter público y colectivo. Una acción de resistencia secreta, discreta, socialmente invisible es moral pero no política. Falta el carácter de declaración pública y de reivindicación. Una acción individual tampoco es política, pues le falta el poder que supone la colaboración, la unión de las voluntades. Sería un error creer acá que la acción política tiene que oponerse a las injusticias extraordinarias, las que son particularmente escandalosas. Al contrario, se trata de hacer visible y de hacerse cargo de la injusticia ordinaria precisamente porque es estructural y, aplicada a nuestra situación contemporánea de crisis ecológica, nos encamina hacia la catástrofe generalizada.

Pero, ¿cómo se puede entender mejor la articulación entre el poder de iniciativa de los sujetos y el carácter de constricción social que el sujeto experimenta? ¿Cómo se puede otorgar un sentido a nuestra responsabilidad si es el sistema, es decir nadie, que produce la injusticia? Vuelve acá la cuestión del estatuto del sujeto de responsabilidad cuando el origen de la injusticia se encuentra a nivel sistémico. Para dilucidar este punto, Young se refiere acá a la noción de práctico-inerte en Jean-Paul Sartre (YOUNG, 2013, p. 53-54) que nos recuerda los análisis anteriores de Jonas. Los agentes sociales persiguen fines conscientes. Sin embargo, la acumulación de los efectos de sus acciones puede producir consecuencias no deseadas que, luego, operan de forma casi-objetiva o mecánica sobre la destinación de los agentes. Piensen por ejemplo al calentamiento global como resultado de un conjunto de efectos de acciones que buscan otra meta que este calentamiento pero que, a su vez hacen que éste pese sobre nosotros como si fuese una fatalidad. El hecho de que el resultado tenga la forma de una constricción casi objetiva no debe hacernos olvidar el origen de este proceso

\footnotetext{
${ }^{1}$ Es una tesis que aleja Young de Arendt para la cual había que distinguir de forma estricta lo socioeconómico de lo político.
} 
que se encuentra en decisiones individuales. Este recuerdo es la condición para hacernos adoptar un punto de vista más estructural sobre nuestras decisiones y acciones con el fin de ver que éstas pueden producir un efecto de necesidad no deseado.

Como se lo puede apreciar, se trata acá da una mirada orientada hacia el futuro, hacia lo que queda por hacer con miras, por ejemplo, a las generaciones futuras y la naturaleza. Es cierto que la perspectiva de cambio abierta por la toma de responsabilidad política no puede garantizar ningún éxito. Pero esta falta de garantía no significa que prevalga un determinismo socio-económico, sino solamente que la posibilidad de cambio queda reducida a una mera posibilidad abierta a contingencias, a decisiones individuales, a valentías personales, a cristalizaciones sociales que pueden ocurrir o no. Pero basta con una posibilidad de cambio para generar las iniciativas de cambio y ofrecer a los agentes el espacio de un "quizás." Dicho de otro modo, no se puede argüir a partir de la rareza de los cambios sociales para desalentar la acción política en nombre de creencias fatalistas. La acción, por ser rara, no deja de ser profunda y puede afectar de forma radical la organización social para que ella, en efecto, sea menos injusta.

Se podría llamar solidaridad a esta responsabilidad social en la medida en que no descansa en la postulación de una pura generosidad del agente o en una cultura de la empatía. Lo que importa acá es la capacidad de reconocer el carácter relacional de mi ser y mi responsabilidad personal en la reactualización de una situación de injusticia. Por ello, la responsabilidad debe ser comprendida como compartida más que como colectiva. Cada uno tiene que tomar su responsabilidad al reconocer que la tiene en lugar de creer que es la colectividad en general que la tiene sin que nadie en particular se la atribuya (YOUNG, 2013, p. 110).

No obstante, existe una tentación de evadir la toma de responsabilidad política a pesar de ser, o bien precisamente porque estamos, en una situación en la cual siempre tenemos una responsabilidad. De hecho, por el solo hecho de actuar conforme a nuestra posición social, colaboramos con la perpetuación de una estructura injusta. Por ello, podemos sucumbir a la tentación de denegar nuestra responsabilidad, de hacernos ciegos a ella. Este escenario se produce cada vez que damos nuestra adhesión a la creencia en la cosificación del mundo social, cuando desvinculamos un efecto social de la cadena causal que lo produce y de la cual formamos parte, cuando no tomamos ninguna responsabilidad bajo el pretexto de que las exigencias de la responsabilidad son infinitas (excesivas), nos 
superan pues apuntan a todas las víctimas del sistema o cuando afirmamos que no nos compete pues es la función del gobierno ${ }^{2}$. Entonces, según Young, existen varias estrategias para huir de su responsabilidad y desconocerse como agente responsable de la totalidad social (YOUNG, 2013, p. 153-170).

Pero reconocerse responsable de la reproducción de un sistema injusto podría desembocar en una culpabilización esterilizante que encierra el sujeto en un pasado originario que lo inscribe, para siempre, en la culpa. Sin embargo, no es necesariamente el caso según Young. El reconocimiento del peso de la responsabilidad no tiene que provocar resentimiento o generar una postura defensiva. Invita más bien a descargarse de esta responsabilidad al tomarla a través de la acción pública y colaborativa. Además, los primeros agentes del cambio social no tienen necesariamente que ser los que ocupan una posición social privilegiada. Al contrario, se puede suponer que de ellos provendrá la mayor resistencia al cambio. Es sobre ellos que la presión social se debe ejercer. La teoría social de Young no elimina los conflictos y la dimensión agonal de la acción bajo una visión romántica y generosa de la realidad social. Al contrario, son las víctimas del sistema quienes pueden iniciar un cambio social; una modificación estructural porque son ellos quienes tienen intereses en el cambio.

Por supuesto, esta esperanza supone desmitologizar la creencia en la cosificación de la acción. Si bien, como lo hemos visto, efectos mecánicos pueden presionarnos en consecuencia de la acumulación de decisiones individuales que tenían otras metas, conviene proceder a una descosificación de estos efectos al adoptar una mirada estructural sobre la acción para identificar cómo nuestras acciones pueden producir estos efectos de necesidad y evitarlos.

Se podría tener la impresión de que la teoría social de Young nos aleja de las preocupaciones por las generaciones futuras y la naturaleza que fueron nuestro punto de partida. No obstante, me parece necesario recordar, en primer lugar, que la situación en la cual el sujeto está inscrito es una situación que no tiene solamente una dimensión social y económica sino también una dimensión ambiental. Como decimos en Chile, el pertenecer o no a zonas de sacrificio (TIRONI, 2018). Es así como las injusticias estructurales atañen

\footnotetext{
2 Young presenta varios argumentos para atender a estas objeciones u obstáculos a la responsabilidad (YOUNG, 2013, p. 153-170).
} 
también a desigualdades medioambientales (HERVE, 2015; LARRERE, 2015) que afectan a las poblaciones. Pero son también situaciones en las que tal o cual especie o tal o cual ecosistema pueden ser dañados. Por supuesto, ellos no son agentes responsables. Pero son objetos de responsabilidad cuya preservación depende de nuestro actuar. Como tales dependen de nuestra responsabilidad política. Solo la acción pública y concertada puede asegurar su preservación.

En segundo lugar, si bien escapamos así de una lectura estrechamente antropocéntrica de la responsabilidad política, queda por preguntar si esta lectura de lo social hace posible una responsabilidad hacia las generaciones futuras que todavía no son, pues son por nacer y advenir. Sin poder entrar detalladamente ahora en esta discusión, se podría hacer valer que la teoría de la responsabilidad política de Young agiliza el desarrollo de una sociedad que se hace responsable de su devenir eco-ambiental, de su sustentabilidad de manera tal que este devenir haga posible la mantención y la promoción, de manera dinámica, del sentido de responsabilidad hacia la totalidad social. Efectivamente, en primer lugar, es necesario reconocer que la responsabilidad universal por las generaciones futuras adopta la forma concreta de la responsabilidad política de una sociedad particular hacia sí misma, hacia su desarrollo de manera que su existencia no sea comprometida, o puesta en peligro, por la radicalización de las injusticias y de la irresponsabilidad. En segundo lugar, esta responsabilidad de una sociedad en particular no puede desconocer la red de relaciones que ella tiene con las otras sociedades y de la cual depende su sustentabilidad propia. El mundo común presupone esta red de relaciones. Por lo tanto, la mantención de las sociedades depende de una responsabilidad compartida que es la forma concreta que toma la responsabilidad hacia las generaciones futuras en general. Es así como se podría entender cómo nuestra especie puede ser considerada capaz de responsabilidad hacia su devenir al no comprometer la posibilidad de su existencia como humanidad.

Para concluir, podríamos formular una última duda. ¿No queda en esta representación de la dinámica social un resto de idealismo? ¿Cómo se podría contar con la organización espontánea de las iniciativas individuales para remediar las injusticias estructurales? ¿No compete a las instituciones asegurarse de la promoción de la justicia? ¿Cómo se podría desarrollar las competencias políticas del ciudadano sin instituciones que procedan de manera coherente con la evidencia que comprende los mecanismos que generan injusticias? ¿Cómo, sin ellas, se podrían tomar medidas, iniciativas que obligan los 
actores sociales a actuar de forma justa incluso cuando este actuar va a contra de sus intereses privados? ¿Cómo se podría prescindir de instituciones internacionales a cargo de la lucha contra las desigualdades ambientales? ¿No hay que reconocer que la necesidad de las instituciones se hace sentir precisamente porque nadie en particular puede asumir la responsabilidad del mal sino la colectividad? Pero, no se trata acá, creo, de pensar una organización social sin instituciones. El punto es más bien de recordar, en primer lugar, que el cambio social puede iniciarse e incluso a veces mantenerse sin instituciones, en segundo lugar, que las metas y el buen funcionamiento de una institución depende, a mediano plazo y en última instancia, de la adhesión de la sociedad a esta institución (YOUNG, 2013, p. 112).

\section{Referencias}

CHAKRABARTY, D. "Changement climatique et capitalisme». Esprit, Paris, n. 441, p. 153-178, 2018.

CHÂTELET, F. «Notes», Manifeste du Parti communiste (K. Marx). Paris : Bordas, 1986.

CRUTZEN, P. “Geology of Mankind”. Nature, v. 415, n. 6867, 2002.

DAVID, C., RÖPCKE, D. «Gűnther Anders, Hans Jonas et les antinomies de l'écologie politique ». Presses de Sciences Po, n. 29, 2004.

GUILLIBERT, P.; HABER, S. Marxisme, études environnementales, approches globales : de nouveaux horizons. In: Marxismes écologiques. Actuel Marx, n. 61, PUF, 2017. p. 13-23.

HERVE, D. Justicia ambiental y recursos naturales. Valparaiso: Ediciones Universitarias de Valparaiso, 2015.

JONAS, H. Técnica, medicina y ética. Barcelona : Paidós, 1997.

JONAS, H. Le phénomène de la vie. Bruxelles : DeBoeck Université, 2001.

JONAS, H. Das Prinzip Verantwortung. Frankfurt am Main: Suhrkamp, 1984.

LARRERE, C. Penser et agir avec la nature. Une enquête philosophique. Paris : éditions la découverte, 2015.

MALM, A. L'anthropocène contre l'bistoire. Le réchauffement climatique. Paris: La fabrique éditions, 2017.

MARX, K. Idéologie allemande. Paris: Editions Sociales, 1966.

MERLEAU-PONTY, M. "La querelle de l'existentialisme", Sens et non-sens. Paris : éditions Nagel, 1965. 
MOORE, J. La nature dans les limites du capital (et vice versa). In: Marxismes écologiques. Actuel Marx, nº1, PUF, 2017. p. 24-46.

OLIVEIRA, J.; POMMIER, E. Vocabulário Hans Jonas. Caxias do Sul: Editora da Universidade de Caxias do Sul, 2019.

POMMIER, E. Ontologie de la vie et éthique de la responsabilité selon Hans Jonas. Paris : Vrin, 2013.

TIRONI, M. Vidas toxicas. In: El Mostrador, 2018. Disponible en: https://www.elmostrador.cl/noticias/opinion/columnas/2018/08/31/vidas-toxicas/.

ULLOA, A. La confrontation d'un citoyen zéro carbone déterritorialisé au sein d'une nature carboné locale-mondiale. In : BEAU, R.; LARRERE, C. Penser l'anthropocène. Paris : SciencesPo Les Presses, 2018.

YOUNG, I. M. Responsibility for Justice. Oxford: Oxford University Press, 2013.

RECEBIDO: $11 / 01 / 2020$

APROVADO: 17/09/2020

RECEIVED: 01/11/2020

APPROVED: 09/17/2020 